\title{
Contrasting lattice geometry dependent versus independent quantities: Ramifications for Berry curvature, energy gaps, and dynamics
}

\author{
Steven H. Simon ${ }^{1}$ and Mark S. Rudner $\circledast^{2}$ \\ ${ }^{1}$ Rudolf Peierls Centre, Oxford University, Oxford OX1 3NP, United Kingdom \\ ${ }^{2}$ Center for Quantum Devices and Niels Bohr International Academy, Copenhagen University, 2100 Copenhagen, Denmark
}

(Received 10 August 2020; revised 2 October 2020; accepted 2 October 2020; published 26 October 2020)

\begin{abstract}
In the tight-binding description of electronic, photonic, or cold atomic dynamics in a periodic lattice potential, particle motion is described in terms of hopping amplitudes and potentials on an abstract network of discrete sites corresponding to physical orbitals in the lattice. The physical attributes of the orbitals, including their locations in three-dimensional space, are independent pieces of information. In this paper we identify a notion of geometry independence: any physical quantity or observable that depends only on the tight-binding parameters (and not on the explicit information about the orbital geometry) is said to be "geometry independent." The band structure itself and, for example, the Chern numbers of the bands in a two-dimensional system, are geometry independent, while the Bloch-band Berry curvature is geometry dependent. Careful identification of geometry dependent versus independent quantities can be used as a novel principle for constraining a variety of results. By extending the notion of geometry independence to certain classes of interacting systems, where the many-body energy gap is evidently geometry independent, we shed light on a hypothesized relation between many-body energy gaps of fractional Chern insulators and the uniformity of Bloch-band Berry curvature in the Brillouin zone. We furthermore explore the geometry dependence of semiclassical wave-packet dynamics, and use this principle to show how two different types of Hall response measurements may give markedly different results due to the fact that one is geometry dependent, while the other is geometry independent. Similar considerations apply for anomalous thermal Hall response, in both electronic and spin systems.
\end{abstract}

DOI: 10.1103/PhysRevB.102.165148

\section{INTRODUCTION}

There has been a recent explosion of interest in the topological properties of noninteracting electronic band structures [1-4]. Of the quantities that have come to the fore during this time, the Berry connection and Berry curvature of the electron bands have played a particularly prominent role. These quantities are not only of importance for understanding the topological nature of the band structure, but they also are crucial in determining the dynamics of particles within the band when electromagnetic perturbations are applied [5].

Often when one studies band structure, one starts with a tight-binding model which defines a set of orbitals $i, j, \ldots$, along with potentials and hopping amplitudes $\left\{t_{i j}\right\}$ between them. These potentials and hopping amplitudes completely define a number of crucial properties of the system, such as its eigenenergies, as well as topological information such as the Chern numbers of its bands. A completely independent piece of information is the set of real-space positions $\left\{\mathbf{r}_{i}\right\}$ of the orbitals. While the eigenenergies of the system are encoded entirely in the hoppings $\left\{t_{i j}\right\}$ and do not depend on

Published by the American Physical Society under the terms of the Creative Commons Attribution 4.0 International license. Further distribution of this work must maintain attribution to the author(s) and the published article's title, journal citation, and DOI. the positions $\left\{\mathbf{r}_{i}\right\}$, quantities such as the Berry curvature do depend explicitly on these positions. We say that the eigenenergies are geometry independent whereas the Berry curvature is geometry dependent. The purpose of this paper is to explore the ramifications of this notion of geometry dependence, in particular for the Berry curvature.

We will be able to apply some of these considerations not only to noninteracting systems, but also to certain interacting models as well. In every case we will consider which quantities depend on geometry independent information, such as hoppings between orbitals, and which quantities depend on geometric information, such as the positions of orbitals. Distinguishing the geometry independent from geometry dependent quantities turns out to give us some very useful and important insight into physical systems and their responses to perturbations.

This paper is organized as follows. In Sec. II we introduce the models that we consider and define the geometry dependent and geometry independent quantities. In Sec. III we focus on the Berry curvature and we show that it is geometry dependent. In Sec. IV we provide our first example of how focusing on geometry dependent versus geometry independent quantities can provide helpful insight and results. Specifically, we consider the widely discussed hypothesis that fractional Chern insulators [6,7], the lattice analogs of fractional quantum Hall systems, should have larger many-body gaps when the Berry curvature is as uniform ("flat") as possible in the Brillouin zone. Since the Berry curvature depends on the detailed 
geometry of the lattice, and under certain conditions (such as pointlike Hubbard interactions) the many-body gaps do not, flat Berry curvature must not be required for obtaining large many-body gaps. Further discussion of this hypothesis and a potential refinement based on our considerations are given in Appendix A.

We then turn in Sec. V to study the dynamical equations of motion of noninteracting particles in a partially filled band in an applied electric field. Since these equations involve the Berry curvature, one might think that a change in geometry (change in the positions of orbitals) may have an effect on the equations of motion. On the other hand, since the tightbinding Hamiltonian defined by $\left\{t_{i j}\right\}$ and the band structure is independent of the geometric information in $\left\{\mathbf{r}_{i}\right\}$, one might expect the dynamics to be completely unchanged by a deformation of $\left\{\mathbf{r}_{i}\right\}$ that leaves $\left\{t_{i j}\right\}$ invariant. In Sec. V we address this issue; we show that the semiclassical wave-packet dynamics are indeed geometry dependent, and discuss the origins and implications of this dependence.

Finally, in Sec. VI we turn our attention to electrical and thermal Hall transport for the case of a partially filled band of noninteracting electrons. For simplicity, we focus on the anomalous case, where no external magnetic field is applied but the system internally breaks time-reversal symmetry and thereby supports nonzero Hall transport coefficients. There are several different experimental configurations which might be used to measure such transport coefficients, which at first examination might be expected to give the same result. On the contrary, we find some configurations give a geometry independent result whereas others give a geometry dependent result. For example, if a physical system with noninteracting fermions has a chemical potential difference applied between two contacts, we obtain a geometry independent Hall response. However, if we couple these fermions to an external electric field and replace the chemical potential difference with a uniform electric field, we instead find a geometry dependent Hall conductivity. We explain this phenomenon in detail in Sec. VI, and discuss how similar considerations can be applied to anomalous thermal Hall transport as well as thermal transport by magnons. We give further calculational details of the Hall response in the disorder-free limit in Appendix B. Note that throughout this work we set $\hbar=1$.

\section{GEOMETRY INDEPENDENCE}

In this section we define the notions of geometry dependence and independence that we explore in this paper. Although it is not necessary to describe crystalline systems in terms of tight-binding models, we will introduce these notions within the tight-binding framework where our results are simplest to discuss. As we discuss in the conclusions, the ideas and results are more general.

To describe electronic states in a crystalline system, we define an orthonormal basis of orbitals $\left\{\left|\alpha, \mathbf{R}+\mathbf{x}_{\alpha}\right\rangle\right\}$, where the label $\alpha$ runs over the different types of orbitals (including spin indices) within the unit cell, $\mathbf{R}$ runs over all lattice vectors, and $\mathbf{x}_{\alpha}$ is the position of orbital $\alpha$ within the unit cell (relative to the unit-cell origin which we take to be at the lattice point $\mathbf{R}$ ). We will always consider the case of multiple orbitals per unit cell. ${ }^{1}$ In the absence of disorder and interactions, the singleparticle band structure of the system can be found from the discrete translationally invariant tight-binding Hamiltonian

$$
H_{0}=\sum_{i j} t_{i j} c_{i}^{\dagger} c_{j}+\text { H.c. }
$$

where $i$ and $j$ each run over all values of $\mathbf{R}$ and $\alpha$, and $c_{i}^{\dagger}$ and $c_{j}$ are creation and annihilation operators for electrons in the corresponding basis modes, respectively. The absolute position of orbital $i$ is given by $\mathbf{r}_{i}=\mathbf{R}+\mathbf{x}_{\alpha}$, for the corresponding values of $\mathbf{R}$ and $\alpha$. These positions are sometimes known as the orbital embeddings [8-10]. The parameters $\left\{t_{i j}\right\}$ encode the information of the tight-binding Hamiltonian $H_{0}$ in a weighted graph (with "onsite" energies represented as the diagonal matrix elements $\left\{t_{i i}\right\}$ ).

Importantly, as we discuss and generalize below, once the tight-binding Hamiltonian has been written in the form of Eq. (1), all of the physical information about the basis orbitals $\left\{\left|\alpha, \mathbf{R}+\mathbf{x}_{\alpha}\right\rangle\right\}$ has been abstracted away. In particular, the graph defined by the matrix elements $\left\{t_{i j}\right\}$ does not explicitly encode the crystal geometry defined by the positions $\left\{\mathbf{r}_{i}\right\}$ of the orbitals. Some physical properties are determined solely by the parameters $\left\{t_{i j}\right\}$, and thus do not explicitly depend on the crystal geometry. We call such properties geometry independent. Other physical properties, as we will see, require knowledge of both the tight-binding parameters and the physical information about the nature of the basis orbitals; we refer to these properties as being geometry dependent. For simplicity, throughout this work we keep the lattice geometry, i.e., the unit-cell size and shape (defined by the lattice vectors $\{\mathbf{R}\}$ ), fixed.

A central example of a geometry independent property is the single-particle band structure itself. The electronic dispersion relation follows directly by diagonalizing $H_{0}$ in Eq. (1), and thereby is determined solely by the values of the tightbinding parameters $\left\{t_{i j}\right\}$. By diagonalizing $H_{0}$, we also obtain the coefficients of the corresponding eigenvectors $\left\{\left|\psi_{n}(\mathbf{k})\right\rangle\right\}$ directly from the parameters $\left\{t_{i j}\right\}$. Here $n$ is a band index, and $\mathbf{k}$ is the wave vector (crystal momentum). Expanding $\left|\psi_{n}(\mathbf{k})\right\rangle$ in the orbital basis $\left\{\left|\alpha, \mathbf{R}+\mathbf{x}_{\alpha}\right\rangle\right\}$ as

$$
\left|\psi_{n}(\mathbf{k})\right\rangle=\sum_{\mathbf{R}, \alpha} e^{i \mathbf{k} \cdot \mathbf{R}} \psi_{n \alpha}(\mathbf{k})\left|\alpha, \mathbf{R}+\mathbf{x}_{\alpha}\right\rangle,
$$

we see that the amplitudes $\psi_{n \alpha}(\mathbf{k})$ (or, equivalently, the eigenstate wave-function amplitudes on all of the sites) are in fact geometry independent, i.e., they are determined solely by $\left\{t_{i j}\right\}$ and do not depend explicitly on the orbital positions $\left\{\mathbf{x}_{\alpha}\right\}$.

To see an example of a geometry dependent quantity, we turn to Bloch's theorem, which is formulated in the coordinate space of the physical system (not simply on the graph $t_{i j}$ ). According to Bloch's theorem, the single-particle eigenstates of an electron in the periodic potential of a crystal lattice can

\footnotetext{
${ }^{1}$ The case where there is only a single orbital per unit cell has zero Berry curvature. Therefore, we focus on the more interesting case of multiple orbitals.
} 
be decomposed in the form

$$
\left|\psi_{n}(\mathbf{k})\right\rangle=e^{i \mathbf{k} \cdot \hat{\mathbf{r}}}\left|u_{n}(\mathbf{k})\right\rangle,
$$

where $\hat{\mathbf{r}}$ is the position operator, $n$ is the band index, and $\left|u_{n}(\mathbf{k})\right\rangle$ is the periodic Bloch function. Importantly, in the position representation, $\left|u_{n}(\mathbf{k})\right\rangle$ exhibits the same periodicity as the lattice.

Through the definition of the periodic Bloch function $\left|u_{n}(\mathbf{k})\right\rangle$ in Eq. (3), the amplitudes $\left\{u_{n \alpha}(\mathbf{k})\right\}$ in

$$
\left|u_{n}(\mathbf{k})\right\rangle=\sum_{\mathbf{R}, \alpha} u_{n \alpha}(\mathbf{k})\left|\alpha, \mathbf{R}+\mathbf{x}_{\alpha}\right\rangle
$$

must depend explicitly on the positions $\left\{\mathbf{x}_{\alpha}\right\}$ of the orbitals; these amplitudes are therefore geometry dependent. Specifically, if we let $\hat{\mathbf{r}}\left|\alpha, \mathbf{R}+\mathbf{x}_{\alpha}\right\rangle \approx\left(\mathbf{R}+\mathbf{x}_{\alpha}\right)\left|\alpha, \mathbf{R}+\mathbf{x}_{\alpha}\right\rangle$, then Eqs. (2)-(4) imply

$$
u_{n \alpha}(\mathbf{k})=e^{-i \mathbf{k} \cdot \mathbf{x}_{\alpha}} \psi_{n \alpha}(\mathbf{k}) .
$$

Thus, we see that, while $\psi_{n \alpha}(\mathbf{k})$ can be determined directly from $H_{0}$, the amplitudes $\left\{u_{n \alpha}(\mathbf{k})\right\}$ require knowledge both of the tight-binding matrix elements and the physical natures of the corresponding orbitals.

Note that in writing Eq. (5) via the approximation $\hat{\mathbf{r}} \mid \alpha, \mathbf{R}+$ $\left.\mathbf{x}_{\alpha}\right\rangle \approx\left(\mathbf{R}+\mathbf{x}_{\alpha}\right)\left|\alpha, \mathbf{R}+\mathbf{x}_{\alpha}\right\rangle$, we consider the orbitals to be pointlike in space; more generally, off-diagonal matrix elements $\left\langle\alpha^{\prime}, \mathbf{R}^{\prime}+\mathbf{x}_{\alpha^{\prime}}|\hat{\mathbf{r}}| \alpha, \mathbf{R}+\mathbf{x}_{\alpha}\right\rangle \neq 0$ may also appear. For well-separated orbitals, these off-diagonal matrix elements are expected to be small. When multiple orbitals on the same atom are involved, the off-diagonal contributions may become more significant. While the considerations below can be extended to include these off-diagonal contributions in the Berry connection, for simplicity we restrict our discussion to the limit of pointlike orbitals where Eq. (5) holds. These contributions may change quantitative results, but do not change our qualitative considerations and conclusions.

Our goal in this work is to characterize the geometry dependence of various physical properties of crystalline systems, analogously to the discussion above. Of particular relevance, we will explore the geometry dependence of the Bloch-band Berry curvature and corresponding consequences for transport. For generality, we will extend the setting beyond the simple discrete translation-invariant quadratic Hamiltonian $H_{0}$ in Eq. (1). In particular, we may consider the role of disorder ${ }^{2}$ (both in hopping and potential), described in tight-binding form via

$$
H_{\mathrm{dis}}=\sum_{i j} \delta t_{i j} c_{i}^{\dagger} c_{j}+\text { H.c. }
$$

\footnotetext{
${ }^{2}$ One may also consider disorder in the position of orbitals without changing any of the onsite potentials, the hoppings, or the interactions between orbitals. This would be a purely geometric change which does not alter the Hamiltonian. However, as emphasized in Sec. V, such a change in position would change the coupling to an externally applied electric field.
}

We may further consider a general extended Hubbard-type interaction term ${ }^{3}$

$$
H_{\text {int }}=\sum_{i, j} U_{i j} n_{i} n_{j}
$$

between electrons in orbitals $i$ and $j$, where $n_{i}=c_{i}^{\dagger} c_{i}$ is the number of particles in orbital $i$. As discussed above for $H_{0}$, the geometry of the crystal is abstracted away from the parameters $\left\{\delta t_{i j}\right\}$ and $\left\{U_{i j}\right\}$; we therefore extend the definition of geometry independence to characterize any physical quantities that are determined solely by the values of $\left\{t_{i j}, \delta t_{i j}, U_{i j}\right\}$ with no explicit input about the geometry or physical nature of the corresponding orbitals/basis states.

\section{GEOMETRIC TRANSFORMATION OF BERRY CURVATURE}

In this section we discuss the geometry dependence of the Bloch-band Berry curvature by examining its explicit dependence on the positions $\left\{\mathbf{x}_{\alpha}\right\}$ of the orbitals as introduced in Sec. II. For now we consider the case of discrete translationinvariant, noninteracting systems, with $H_{\text {dis }}=0$ and $H_{\text {int }}=$ 0 . Using the periodic Bloch functions $\left\{\left|u_{n}(\mathbf{k})\right\rangle\right\}$ defined via Eqs. (3) and (4), we define the Berry connection of the $n$th band as

$$
\mathcal{A}_{n}(\mathbf{k})=i\left\langle u_{n}(\mathbf{k})\left|\nabla_{\mathbf{k}}\right| u_{n}(\mathbf{k})\right\rangle .
$$

The corresponding Berry curvature is given by [5]

$$
\boldsymbol{\Omega}_{n}(\mathbf{k})=\nabla_{\mathbf{k}} \times \mathcal{A}_{n}(\mathbf{k})
$$

We now investigate the geometry dependence of $\mathcal{A}_{n}(\mathbf{k})$ and $\boldsymbol{\Omega}_{n}(\mathbf{k})$. Consider two systems, (1) and (2), described by the same tight-binding parameters $\left\{t_{i j}\right\}$ in Hamiltonian $H_{0}$, in Eq. (1). The physical details of the systems may be different, however, with orbitals $\{\alpha\}$ being of possibly different types, at positions $\left\{\mathbf{x}_{\alpha}^{(1)}\right\}$ and $\left\{\mathbf{x}_{\alpha}^{(2)}\right\}$. As discussed in the previous section, systems (1) and (2) will exhibit identical band structures. As we now show, the Berry connections and Berry curvatures in general depend on the details of $\left\{\mathbf{x}_{\alpha}^{(1)}\right\}$ and $\left\{\mathbf{x}_{\alpha}^{(2)}\right\}$.

To characterize the relationship between the Berry connections and curvatures of systems (1) and (2), for each orbital $\alpha$ we define the relative displacement

$$
\delta \mathbf{x}_{\alpha} \equiv \mathbf{x}_{\alpha}^{(2)}-\mathbf{x}_{\alpha}^{(1)}
$$

to characterize the relative shift of orbital $\alpha$ between the two structures. ${ }^{4}$ As argued above, the wave-function amplitudes

\footnotetext{
${ }^{3}$ More generally, the interaction may involve four orbitals, through terms of the form $\sum_{i j k l} V_{i j k l} c_{i}^{\dagger} c_{j}^{\dagger} c_{k} c_{l}$. However, for our discussion of geometry independent properties, the Hubbard-type interaction that we consider in Eq. (7) is most relevant.

${ }^{4}$ The special case $\delta \mathbf{x}_{\alpha}=\delta \mathbf{x}$ independent of $\alpha$ corresponds to uniform translation of the entire sample. Here we focus on the more interesting case where different orbitals are allowed to shift independently.
} 
$\left\{\psi_{n \alpha}(\mathbf{k})\right\}$ in Eq. (2) must be the same for systems (1) and (2). However, according to Eq. (5), within the approximation of pointlike orbitals, the amplitudes $u_{n \alpha}^{(1)}(\mathbf{k})$ and $u_{n \alpha}^{(2)}(\mathbf{k})$ of the corresponding periodic Bloch functions must be related by

$$
u_{n \alpha}^{(2)}(\mathbf{k})=e^{-i \mathbf{k} \cdot \delta \mathbf{x}_{\alpha}} u_{n \alpha}^{(1)}(\mathbf{k}) .
$$

In principle, there could also be a relative gauge transformation $e^{i \chi(\mathbf{k})}$ on the right-hand side of Eq. (11), where $\chi(\mathbf{k})$ is an ( $\alpha$-independent) arbitrary single-valued function over the Brillouin zone. Here we ignore this possibility; we will further discuss this particular gauge choice near Eq. (22). The corresponding Berry connections are related via

$$
\mathcal{A}_{n}^{(2)}(\mathbf{k})=\mathcal{A}_{n}^{(1)}(\mathbf{k})+\overline{\delta \mathbf{x}_{n}}(\mathbf{k}),
$$

where

$$
\overline{\delta \mathbf{x}_{n}}(\mathbf{k}) \equiv \sum_{\alpha} \delta \mathbf{x}_{\alpha}\left|u_{n \alpha}(\mathbf{k})\right|^{2} .
$$

Here, $\overline{\delta \mathbf{x}_{n}}(\mathbf{k})$ may be interpreted as a $\mathbf{k}$-dependent shift of the electron position within the unit cell. The corresponding Berry curvature then becomes

$$
\boldsymbol{\Omega}_{n}^{(2)}(\mathbf{k})=\boldsymbol{\Omega}_{n}^{(1)}(\mathbf{k})+\nabla_{\mathbf{k}} \times \overline{\delta \mathbf{x}_{n}}(\mathbf{k}) .
$$

This relation has been long known to experts (see, for example, Refs. [10,11] and the Supplemental Material of Ref. [12]). As discussed in Refs. [10,13,14], this type of transformation on the orbital positions corresponds to a unitary transformation on the Bloch Hamiltonian $H(\mathbf{k})$.

We note that topological properties, such as the Chern number $C_{n}$ of band $n$ in a two-dimensional system, are geometry independent: through its definition as an integral over the entire (periodic) two-dimensional Brillouin zone (BZ),

$$
C_{n}=\frac{1}{2 \pi} \int_{\mathrm{BZ}} d^{2} k \Omega_{n}(\mathbf{k}),
$$

we see that the contribution of the second term in Eq. (14) vanishes by Stokes' theorem. ${ }^{5}$ Similarly, for three-dimensional systems the Berry flux through a fixed surface in $k$ space,

$$
C=\frac{1}{2 \pi} \int \mathbf{d} \mathbf{S} \cdot \boldsymbol{\Omega}_{n}(\mathbf{k})
$$

is geometry independent. Thus, for example, if there is a Weyl node carrying a monopole of Berry flux, this flux must be the same for any systems with identical tight-binding parameters, independent of the geometric information about atomic coordinates. Note, however, that an integral of the Berry curvature over a domain with boundary is not generally geometry independent.

\section{GEOMETRIC STABILITY HYPOTHESIS}

In the many-body context, bands with nontrivial Berry curvature play a central role, for example, in the fractional quantum Hall effect, which occurs when Landau levels are

\footnotetext{
${ }^{5}$ Stokes' theorem applies here since $\overline{\delta \mathbf{x}_{n}}(\mathbf{k})$ is continuous, single
} valued, and nonsingular over the Brillouin zone. partially filled with interacting particles. Landau levels, which are dispersionless and feature a uniform Berry curvature throughout the Brillouin zone, are obtained when a free electron is subjected to a uniform magnetic field. In recent years, considerable interest has grown in the study of analogous phases known as fractional Chern insulators [6,7,15-23], which may arise in partially filled, topologically nontrivial bands of interacting particles on a two-dimensional lattice. By analogy to the Landau levels that underlie the fractional quantum Hall effect, it has long been hypothesized that stable (i.e., large gap) fractionally filled states on the lattice most favorably arise when the Berry curvature is uniform in the Brillouin zone [6,7,12,20,21,23-28]. In a slightly more general form (see Ref. [12] and Appendix A), this hypothesis has been called a "geometric stability hypothesis." As we now discuss (see also Ref. [11]), based on the notions of geometry dependence and independence formulated above, this hypothesis is not well defined as stated.

Note that, as our aim in this paper is to highlight the notions of geometry dependence and independence defined in Sec. II and to demonstrate how they may be used to obtain new perspectives on various physical phenomena, an extended discussion of the geometric stability hypothesis is beyond our scope. However, for context, in Appendix A we provide a more detailed summary of the proposed conjectured relations between many-body gaps and quantities that characterize the geometry of a system's Bloch bands.

Consider a two-dimensional system with a Hamiltonian as defined in Eqs. (1) and (7), and partial filling of the highest occupied band. For example, we may consider a nearest-neighbor hopping Hamiltonian on some lattice, with an onsite Hubbard interaction. With these definitions, the Hamiltonian is geometry independent in the sense defined in Sec. II (making no reference to the positions of orbitals). Hence, the many-body gap is entirely independent of changes in geometry so long as the potentials, hopping matrix elements, and interactions are kept fixed. However, under this change in geometry the Berry curvature (and hence its flatness over the Brillouin zone) changes according to Eqs. (10) and (14).

As the arguments above show, the flatness (or uniformity) of the Berry curvature can be tuned independently of the many-body gap; this seemingly contradicts the idea of a simple geometric stability hypothesis $[11,29]$. Indeed, this conundrum was also noted in the Supplemental Material of Ref. [12], where it was suggested that certain positions in the unit cell are more physical than others. On the other hand, one may justifiably study many different physical systems with different geometries (yet equal many-body gaps), with none being particularly more valid than any other. In Appendix A we offer an alternative formulation of the conjecture on the stability of fractional Chern insulator phases that takes into account the special role of the geometries identified in Ref. [12] and in numerical studies [12,23,27], as well as the notions of geometry dependence formulated in this work. We emphasize that a further investigation of the stability of fractional Chern insulators is beyond the scope of this work; our intention here is to highlight the utility of the notion of geometry independence and to use it to suggest a direction for future work. 


\section{SEMICLASSICAL DYNAMICS}

In this section we consider the semiclassical dynamics of wave packets for noninteracting particles. It is well known that Berry curvature, which is geometry dependent as shown in Eq. (14), is relevant to semiclassical dynamics. Here we seek to elucidate the origin of this geometry dependence. In this section we will consider a geometry independent hopping Hamiltonian as in Eq. (1), and neglect interparticle interactions. Crucially, to determine the equations of motion, we must allow the particles in the model to also be coupled to a uniform externally applied electric field $\mathbf{E}(\mathbf{r})=-\nabla \phi(\mathbf{r})$. The coupling to the externally applied potential, given the assumption of pointlike orbitals (see Sec. II), can be written as

$$
H_{\mathrm{ext}}=\sum_{i} \phi\left(\mathbf{r}_{i}\right) n_{i}
$$

where $n_{i}=c_{i}^{\dagger} c_{i}$ is the occupation-number operator for orbital $i$ and $\mathbf{r}_{i}$ is the position of orbital $i$. Note that this coupling depends explicitly on the geometry, in particular, it depends on the orbitals' positions.

\section{A. Berry curvature and semiclassical dynamics}

Considering wave packets in a Bloch-band structure, one can determine a set of semiclassical equations of motion. The full derivation of such equations (and extensive discussion) is given in Refs. [5,30], for example. Here we will repeat just a few key pieces of the discussion.

Using a general construction (see, e.g., Sec. IV A of Ref. [5]), we form a wave packet $|W(t)\rangle$ in band $n$ as

$$
|W(t)\rangle=\int d \mathbf{k} w(\mathbf{k}, t)\left|\psi_{n}(\mathbf{k})\right\rangle,
$$

where $\left|\psi_{n}(\mathbf{k})\right\rangle$ is the full Bloch wave function for the $n$th band (including the plane-wave part) [see Eq. (2)]. The amplitudes $w(\mathbf{k}, t)$ describe the (time-dependent) structure of the wave packet. We consider wave packets that are tightly localized in reciprocal space, such that $|w(\mathbf{k}, t)|^{2}$ is approximately distributed like a delta function centered at wave vector $\mathbf{k}_{c}$ (which may be a function of time):

$$
\mathbf{k}_{c}(t)=\int d \mathbf{k} \mathbf{k}|w(\mathbf{k}, t)|^{2}
$$

The real-space position of the wave packet is determined by the phases of the complex amplitudes $w(\mathbf{k}, t)$, as well as the structure of the wave-function components $\left|\psi_{n}(\mathbf{k})\right\rangle$ and the orbital positions $\left\{\mathbf{x}_{\alpha}\right\}$. As discussed in Refs. [5,30], the average position of the wave packet can be written as

$$
\mathbf{r}_{c}(t)=\mathbf{F}[w(\mathbf{k}, t)]+\mathcal{A}_{n}\left(\mathbf{k}_{c}\right),
$$

where $\mathcal{A}_{n}\left(\mathbf{k}_{c}\right)$ is the Berry connection for the band [Eq. (8)] and (suppressing time arguments)

$$
\mathbf{F}[w(\mathbf{k})]=\int d \mathbf{k} w^{*}(\mathbf{k})\left(i \nabla_{\mathbf{k}}\right) w(\mathbf{k})=-\left.\nabla_{\mathbf{k}} \arg w(\mathbf{k})\right|_{\mathbf{k}=\mathbf{k}_{c}} .
$$

From here on we will drop the subscript $c$, such that $\mathbf{r}$ and $\mathbf{k}$ will describe the center-of-mass position and wave vector of the wave packet.
As discussed in Ref. [5], in the presence of a weak electric field, the center-of-mass position and momentum of the wave packet in band $n$ (assuming nondegenerate bands ${ }^{6}$ ) evolve according to the semiclassical equations of motion

$$
\begin{gathered}
\dot{\mathbf{r}}=\nabla_{\mathbf{k}} \varepsilon_{n}(\mathbf{k})-\dot{\mathbf{k}} \times \boldsymbol{\Omega}_{n}(\mathbf{k}), \\
\dot{\mathbf{k}}=-e \mathbf{E},
\end{gathered}
$$

where $\varepsilon_{n}(\mathbf{k})$ and $\boldsymbol{\Omega}_{n}(\mathbf{k})$ are the band structure dispersion and Berry curvature [see Eq. (9)] for band $n$, and $\mathbf{E}$ is the externally applied electric field. Wave-packet dynamics governed by these equations have been investigated recently in cold atoms [31-36]. Note that we have assumed zero external magnetic field, although we will generally assume that timereversal symmetry is broken so that one can have a nonzero (anomalous) Hall response. Below, we will drop the bandindex subscript $n$ on $\varepsilon_{n}(\mathbf{k})$ and $\boldsymbol{\Omega}_{n}(\mathbf{k})$.

\section{B. Role of orbital positions in the unit cell}

We are now ready to compare the equations of motion for wave packets in two systems (1) and (2), described by the same tight-binding parameters $\left\{t_{i j}\right\}$ but different physical geometries (i.e., atomic positions $\left\{\mathbf{r}_{i}\right\}$ ), as discussed in Sec. III. Given that the tight-binding Hamiltonians for systems (1) and (2) are identical, we may pick gauges for each such that the wave-function amplitudes of the eigenstates $\psi_{n \alpha}^{(1)}(\mathbf{k})$ and $\psi_{n \alpha}^{(2)}(\mathbf{k})$ are identical in the site basis. With this choice of gauge, the wave packets formed according to Eq. (16) with identical choices of $w(\mathbf{k})$ will be spatially shifted with respect to one another. Note that specifying a relative gauge for the two systems is necessary in order to make physically meaningful comparisons between wave packets in the two systems. The conclusions below about the equations of motion for wave packets in the two systems are gauge independent.

Using Eq. (12) in Eq. (18), we find the relative shift of center-of-mass position of the wave packet between systems (1) and (2):

$$
\mathbf{r}^{(2)}=\mathbf{r}^{(1)}+\overline{\delta \mathbf{x}}(\mathbf{k}),
$$

where we have dropped the subscript $n$ on $\overline{\delta \mathbf{x}}(\mathbf{k})$ from Eq. (12) since we have focused on a particular band. This expression has a natural physical interpretation in terms of the shift of the electronic wave function in the unit cell [see text below Eq. (13)].

The equations of motion for wave packets in systems (1) and (2) are given by Eqs. (20) and (21). Note that Eq. (21) is identical for the two cases, as it makes no reference to $\mathbf{r}$ or to the Berry curvature. On the other hand, we obtain two different equations of motion from Eq. (20):

$$
\dot{\mathbf{r}}^{(1)}=\nabla_{\mathbf{k}} \varepsilon(\mathbf{k})-\dot{\mathbf{k}} \times \boldsymbol{\Omega}^{(1)}(\mathbf{k})
$$

and

$$
\dot{\mathbf{r}}^{(2)}=\nabla_{\mathbf{k}} \varepsilon(\mathbf{k})-\dot{\mathbf{k}} \times \boldsymbol{\Omega}^{(2)}(\mathbf{k}),
$$

\footnotetext{
${ }^{6}$ In the case of degenerate bands, the equations of motion allow transitions between the degenerate states [5]. If bands are near degenerate, then the nondegenerate equations of motion are applicable only for perturbations weak enough not to mix the states.
} 
where the Berry curvatures $\boldsymbol{\Omega}^{(1)}(\mathbf{k})$ and $\boldsymbol{\Omega}^{(2)}(\mathbf{k})$ are related by Eq. (14).

It is natural to ask whether these two equations describe the same dynamics or not. Since the two systems (in the absence of the applied field) are described by identical tight-binding Hamiltonians, one might expect the same dynamical response. Of course, the shift in position of the orbitals should result in some "trivial" changes in observables due to the change in location where the electrons reside. Beyond this trivial effect, one may ask whether the populations on the sites $\left\langle n_{i}\right\rangle$ evolve identically in the two cases. If the two sets of equations of motion were to describe the same dynamics in this latter sense (i.e., with a simple change of variables to account for the orbital shifts), one would be able to recover Eq. (23) from Eq. (24) by directly substituting the expressions for $\mathbf{r}^{(2)}$ in terms of $\mathbf{r}^{(1)}$ [Eq. (22)] and $\boldsymbol{\Omega}^{(2)}$ in terms of $\boldsymbol{\Omega}^{(1)}$ [Eq. (14)] into Eq. (24). As we now show, performing this substitution does not return Eq. (23); hence, Eqs. (23) and (24) describe different physical dynamics.

To track down the source of this difference, we perform the substitution described above and obtain

$$
\frac{d}{d t}\left[\mathbf{r}^{(1)}+\overline{\delta \mathbf{x}}(\mathbf{k})\right]=\nabla_{\mathbf{k}} \varepsilon(\mathbf{k})-\dot{\mathbf{k}} \times\left[\boldsymbol{\Omega}^{(1)}(\mathbf{k})+\nabla_{\mathbf{k}} \times \overline{\delta \mathbf{x}}(\mathbf{k})\right] .
$$

Evaluating the left-hand side of this expression gives $\frac{d}{d t}\left[\mathbf{r}^{(1)}+\bar{\delta} \mathbf{x}(\mathbf{k})\right]=\dot{\mathbf{r}}^{(1)}+\left(\dot{\mathbf{k}} \cdot \nabla_{\mathbf{k}}\right) \overline{\delta \mathbf{x}}(\mathbf{k})$. Regrouping terms, we obtain

$$
\dot{\mathbf{r}}^{(1)}=\nabla_{\mathbf{k}} \varepsilon(\mathbf{k})-\dot{\mathbf{k}} \times \boldsymbol{\Omega}^{(1)}(\mathbf{k})+\dot{\mathbf{r}}_{\mathrm{extra}}
$$

where

$$
\dot{\mathbf{r}}_{\text {extra }}=-\dot{\mathbf{k}} \times\left[\nabla_{\mathbf{k}} \times \overline{\delta \mathbf{x}}(\mathbf{k})\right]-\left(\dot{\mathbf{k}} \cdot \nabla_{\mathbf{k}}\right) \overline{\delta \mathbf{x}}(\mathbf{k}) .
$$

Thus, we see that the motion of a wave packet in system (2) differs from that of a wave packet in system (1) by the additional term $\dot{\mathbf{r}}_{\text {extra }}$ appearing in Eqs. (25) and (26).

To determine the meaning of this additional term, it is worth simplifying the expression for $\dot{\mathbf{r}}_{\text {extra }}$. Using Eq. (21) to make the replacement $\dot{\mathbf{k}}=-e \mathbf{E}$, along with the vector triple product identity $\mathbf{A} \times[\mathbf{B} \times \mathbf{C}]=\mathbf{B}(\mathbf{A} \cdot \mathbf{C})-(\mathbf{A} \cdot \mathbf{B}) \mathbf{C}$, we obtain

$$
\dot{\mathbf{r}}_{\text {extra }}=\nabla_{\mathbf{k}}[e \mathbf{E} \cdot \overline{\delta \mathbf{x}}(\mathbf{k})] .
$$

What is the origin of the extra term $\dot{\mathbf{r}}_{\text {extra }}$ ? The key is to note that the coupling of the electron to the uniform external electric field is sensitive to the positions of the orbitals. In terms of the corresponding electric potential, we have $\mathbf{E}=$ $-\nabla_{\mathbf{r}} \phi(\mathbf{r})$. The electrostatic potential for the wave packet in system (2) is given by

$$
\begin{aligned}
\phi\left(\mathbf{r}^{(2)}\right) & =\phi\left[\mathbf{r}^{(1)}+\overline{\delta \mathbf{x}}(\mathbf{k})\right] \\
& \approx \phi\left(\mathbf{r}^{(1)}\right)-\mathbf{E} \cdot \overline{\delta \mathbf{x}}(\mathbf{k}) .
\end{aligned}
$$

The energy of the wave packet in system (2) is therefore shifted by a k-dependent energy $\delta \varepsilon(\mathbf{k})$ relative to that of the corresponding wave packet in system (1):

$$
\delta \varepsilon(\mathbf{k})=-e\left[\phi\left(\mathbf{r}^{(2)}\right)-\phi\left(\mathbf{r}^{(1)}\right)\right]=e \mathbf{E} \cdot \overline{\delta \mathbf{x}}(\mathbf{k}) .
$$

In terms of wave-packet propagation, a k-dependent energy shift $\delta \varepsilon(\mathbf{k})$ essentially modifies the dispersion; the corresponding change of group velocity is reflected in the additional contribution to the velocity $\dot{\mathbf{r}}_{\text {extra }}$ in Eq. (27). The difference in the equations of motion for wave packets in the two systems arises from the fact that the electrons in the two systems couple differently to the external field. At the level of the tight-binding Hamiltonian, we note that this difference arises from the fact that the electric potential $\phi(\mathbf{r})$ corresponding to the uniform electric field introduces onsite energies that explicitly depend on the atomic coordinates $\left\{\mathbf{r}_{i}\right\}$, thus breaking the geometry independence of the tight-binding Hamiltonian. Nonetheless, as also pointed out in Ref. [13], Appendix D (and shown explicitly there for a two band model), both before and after the transformation, the system properly satisfies the semiclassical equations of motion (20) and (21).

\section{Lagrangian formulation}

For further insight into the geometry dependence of the semiclassical wave-packet dynamics, it is instructive to investigate the role of the orbital positions $\left\{\mathbf{r}_{i}\right\}$ in the semiclassical phase-space Lagrangian. We begin with the Lagrangian describing the dynamics of a wave packet in band $n$ (in the absence of an applied magnetic field), as given in Eq. (5.7) in Ref. [5]:

$$
L(\mathbf{r}, \mathbf{k}, \dot{\mathbf{r}}, \dot{\mathbf{k}})=\mathbf{k} \cdot \dot{\mathbf{r}}-\varepsilon_{n}(\mathbf{k})+e \phi(\mathbf{r})+\dot{\mathbf{k}} \cdot \mathcal{A}_{n}(\mathbf{k}) .
$$

Note that here $\mathbf{r}$ and $\mathbf{k}$ correspond to $\mathbf{r}_{c}$ and $\mathbf{k}_{c}$, the average position and momentum of the wave packet [see Eqs. (17) and (18)]. Although $\mathbf{r}_{c}$ implicitly depends on $\mathbf{k}_{c}$ via Eq. (18), $\mathbf{r}$ and $\mathbf{k}$ should be treated as independent variables in the Lagrangian [they are in fact independent implicit parameters in the wave function $|W\rangle$ in Eq. (16)]. In particular, $\nabla_{\mathbf{k}} \phi(\mathbf{r})=0$.

Again, comparing the behavior for two systems with identical tight-binding parameters but different atomic positions, the Lagrangian in Eq. (29) can be written as $L^{(s)}=\mathbf{k}^{(s)} \cdot \dot{\mathbf{r}}^{(s)}$ $\varepsilon_{n}\left(\mathbf{k}^{(s)}\right)+e \phi\left(\mathbf{r}^{(s)}\right)+\dot{\mathbf{k}}^{(s)} \cdot \mathcal{A}_{n}^{(s)}\left(\mathbf{k}^{(s)}\right)$ for system $s=1$, 2. Using the coordinate transformation for $\mathbf{r}^{(2)}$ in terms of $\mathbf{r}^{(1)}$ given in Eq. (22), along with its time derivative $\dot{\mathbf{r}}^{(2)}=$ $\dot{\mathbf{r}}^{(1)}+\frac{d}{d t} \bar{\delta} \mathbf{x}(\mathbf{k})$ and the relation between Berry connections in Eq. (12), we express the Lagrangian for system (2) in terms of the coordinates for system (1):

$$
\begin{aligned}
L^{(2)}= & \mathbf{k} \cdot \dot{\mathbf{r}}^{(1)}-\varepsilon(\mathbf{k})+e \phi\left[\mathbf{r}^{(1)}+\overline{\delta \mathbf{x}}(\mathbf{k})\right] \\
& +\dot{\mathbf{k}} \cdot \mathcal{A}^{(1)}(\mathbf{k})+\text { T.D. }
\end{aligned}
$$

where "T.D." stands for "total derivative." In writing Eq. (30) we have used $\mathbf{k}^{(2)}=\mathbf{k}^{(1)} \equiv \mathbf{k}$ and $\dot{\mathbf{k}}^{(2)}=\dot{\mathbf{k}}^{(1)} \equiv \dot{\mathbf{k}}$, along with

$$
\frac{d}{d t}[\mathbf{k} \cdot \overline{\delta \mathbf{x}}(\mathbf{k})]=\mathbf{k} \cdot \frac{d}{d t} \overline{\delta \mathbf{x}}(\mathbf{k})+\dot{\mathbf{k}} \cdot \overline{\delta \mathbf{x}}(\mathbf{k}) .
$$

Dropping the total derivative term, which does not affect the equations of motion, we see that the Lagrangian in Eq. (30) takes the usual form as that in Eq. (29), with one twist: the potential is evaluated at a new $\mathbf{k}$-dependent position. Although ordinarily we do not take $\mathbf{k}$ derivatives of the potential (see discussion above), due to the coordinate transformation that we have taken (where $\mathbf{r}^{(1)}$ does not correspond to the center of the wave packet, and therefore $\dot{\mathbf{r}}^{(1)}$ does not correspond to the wave packet's velocity), we will obtain $-\nabla \phi \cdot \overline{\delta \mathbf{x}}(\mathbf{k})=$ 
$\mathbf{E} \cdot \overline{\delta \mathbf{x}}(\mathbf{k})$ as a correction to the dispersion. This term simply makes up for the bad parametrization of the wave function that we took by using a "coordinate" appropriate for a system with a different geometry, that does not actually correspond to the center of the wave packet in system (2).

\section{ANOMALOUS HALL TRANSPORT}

Having highlighted the importance of the geometry independent versus geometry dependent contributions to semiclassical dynamics, we now turn to examine the Hall effect as an example. We will consider both the electric current response (the usual Hall effect) and the thermal response (the thermal Hall effect, or Righi-Leduc effect). We will assume that the hopping between orbitals generically breaks time reversal. The appearances of nonzero Hall transport coefficients in the absence of an external magnetic field are known as anomalous Hall effects [37]. Our considerations also directly apply to systems with a commensurate externally applied magnetic field, where we absorb the effects of the magnetic field into hopping phases and work with the magnetic unit cell as the elementary unit cell of the system.

We emphasize that in the case of a filled band, for noninteracting (or weakly interacting) fermions, the electrical Hall conductance (and conductivity) will be quantized and is given by $C e^{2} / h$ with $C$ the Chern number. As mentioned in Sec. III, the Chern number is geometry independent. Similarly the Righi-Leduc effect will be quantized in this case. The more interesting case to study, which we will focus on here, is the case of a partially filled band.

The study of anomalous Hall responses is complicated, with several distinct physical processes contributing, and a fair amount of competing claims persisting in the literature (see Refs. [37,38] for a detailed discussion of the competing factors and the subtleties that have historically been the source of confusion). Because of these complexities it is useful to focus on the simple case of a disorder-free system, which clearly demonstrates the distinction between geometry dependent versus geometry independent responses. Although this example is particularly simple to discuss, our main conclusions are not restricted to the disorder-free case.

\section{A. Electric Hall current response}

Consider a noninteracting system described by a tightbinding model as defined in Eq. (1). Below we describe two scenarios for measuring the electric Hall current response (current transverse to the applied bias): (i) by applying a chemical potential difference between two contacts or (ii) by applying a uniform electric field. We assume no coupling to phonons.

\section{Geometry independent response}

Here we consider neutral particles, and imagine attaching the system to two reservoirs which have a chemical potential difference between them. Taking a cylindrical geometry (periodic boundary conditions in the direction perpendicular to the axis connecting the two reservoirs), we consider the total current that flows around the circumference of the cylinder (i.e., perpendicular to the direction between these reservoirs).
This model is completely geometry independent in the sense defined in Sec. III. Nowhere in the Hamiltonian or the driving perturbation do the positions of orbitals in the unit cell explicitly enter. Further, we may add geometry independent disorder (i.e., a random potential in the site basis) and interactions as in Eqs. (6) and (7) and the Hamiltonian still makes no reference to the positions of orbitals. Finally, the observable of interest, i.e., the current that flows around the cylinder, can also be formulated as a geometry independent quantity. ${ }^{7}$ Thus, the response of this system is geometry independent. For reference, in Appendix B 2 we provide an explicit calculation of the current density in the clean limit, and demonstrate that it is indeed geometry independent.

\section{Geometry dependent response}

Instead of applying a chemical potential difference between contacts, here we consider coupling the system to a uniform external electric field as in Eq. (15). This perturbation is explicitly geometry dependent; as shown in Sec. VB, the geometry dependence is crucial for obtaining the correct semiclassical equations of motion.

To be concrete, and for maximal simplicity, we imagine a torus geometry with an electric field directed around one of the handles. This situation can be made more realistic by considering an annulus (Corbino) geometry with electric field directed in the azimuthal direction around the ring. (Such an electric field can be generated by slowly varying a magnetic flux through the hole of the annulus.)

The (linear) current response to the applied electric field is defined to be the conductivity. A well-known result in the literature gives the so-called intrinsic contribution to the anomalous Hall effect as the integral of the Berry curvature $\Omega(\mathbf{k})$ over occupied states [37]. Here we give the result in two dimensions (so $\Omega$ is a scalar) for simplicity,

$$
\sigma_{x y}^{\text {int }}(\mu)=e^{2} \int \frac{d^{2} k}{(2 \pi)^{2}} \Omega(\mathbf{k}) n_{F}[\varepsilon(\mathbf{k})-\mu],
$$

where $n_{F}$ is the Fermi occupation factor. If multiple bands are partially filled, these must be summed over.

This intrinsic response fully describes the Hall conductivity in the absence of interactions and absence of disorder (taking a limit of frequency going to zero, and also taking the limit of very small applied electric field at the same time, see Appendix B 1). One approach to deriving this result is to use the Kubo formula. A more transparent approach is to substitute Eq. (21) into Eq. (20) and integrate the anomalous velocity term over all filled states. In any case, because the Berry curvature is geometry dependent, so is this resultant Hall response.

If we would consider the case with disorder, the calculation of the Hall response would be more complicated but would

\footnotetext{
${ }^{7}$ One can imagine making a cut down the long axis of the cylinder and keeping track of the number of electrons per second that jump across this cut. If we change the geometry of the system, the number of electrons per second jumping across the cut is completely unchanged so long as we do not move any orbitals across the cut. Further, in the dc limit, due to current conservation, the current must be fully independent of where we make the cut.
} 
remain geometry dependent. In particular, as described for example in Refs. [37,38], there are several contributions to the Hall conductivity in addition to the intrinsic part. However, in the limit $\omega \tau \gg 1$ with $\omega \rightarrow 0$, the intrinsic part, Eq. (31), correctly gives the Hall conductivity $[37,39,40]$. Here $\tau$ is the transport (scattering) lifetime. Further, when $\omega$ is strictly zero (at least for weak and smooth disorder) the intrinsic contribution can still be identified as it is the only piece that reflects the Berry curvature deep within the Fermi sea, ${ }^{8}$ with all other pieces only sensitive to the Berry curvature near the Fermi surface [38].

\section{Comparison}

It is worth noting that it is commonly expected that the response of a system to a chemical potential difference is the same as its response to an electric field: both perturbations apply a bias that drives current. However, as we have shown here, in some circumstances, and depending on the precise questions asked, they give different results. One might ask whether the situation is different in the presence of disorder. While disorder makes the calculations of the current response much more complicated, we can nonetheless be assured that the two situations described above, with (i) chemical potential difference and (ii) electric field, will give different Hall current responses, as the former is geometry independent and the latter is generically geometry dependent.

It is interesting to note that in the geometry independent case (i) where we apply a chemical potential difference, the perturbation to the system does not change the Hamiltonian of the system at all, it only changes the chemical potential of the particles in the leads. On the contrary, the applied electric field in case (ii) perturbs the Hamiltonian of the entire system.

\section{B. Thermal Hall current response}

Analogous to the two cases in the last subsection, here we consider a temperature difference or gradient applied in one direction, while the thermal current orthogonal to the gradient is measured. The physics of this heat current response is similar in spirit to that of the electric current response.

\section{Geometry independent response}

Analogous to Sec. VIA 1 we imagine attaching our (disorder-free) system in a cylindrical geometry to two thermal reservoirs which have a temperature difference. We then

\footnotetext{
${ }^{8}$ Note that the Berry flux through the Fermi sea, which controls the Hall response via Eq. (31), can be cast via Stokes' theorem as a Fermi-surface property (equal to the Berry phase acquired by an electron that traverses the Fermi surface) [42]. Nonetheless, this quantity is intimately tied to the geometry of the Bloch wave functions throughout the Fermi sea, whereas the skew-scattering and side-jump contributions to the Hall conductivity that result from scattering off of impurities are sensitive predominantly to the wave functions at the Fermi surface. In particular, for smooth disorder, the side jump contribution is proportional to the Berry curvature at the Fermi surface [57], which can be tuned (even to zero) independently of the Berry flux.
}

measure the heat current perpendicular to the direction between these reservoirs. We assume that there is no coupling to phonons or any other degrees of freedom outside of the system (except coupling to the reservoirs).

Again, we use the same tight-binding model, Eq. (1), which is completely geometry independent. Nowhere in the definition of the model or in the coupling to the reservoirs did we need to specify the position of the orbitals in the unit cell. Again we even may add geometry independent disorder and interactions as in Eqs. (6) and (7), and the heat current that flows around the circumference of the cylinder must remain independent of the geometric information about the orbital positions within the unit cell.

\section{Geometry dependent response}

Analogous to Sec. VI A 2 here we apply a uniform temperature gradient to the system. In other words, at position $\mathbf{r}_{i}$ in the sample there will be a weak coupling to a reservoir with temperature $T\left(\mathbf{r}_{i}\right)$ where $T$ has a uniform gradient. Since the reservoir temperature coupled to orbital $i$ is dependent on the position of orbital $i$, we expect the response to be explicitly geometry dependent.

This thermal Hall conductivity (also known as the RighiLeduc coefficient) can be calculated in several ways. A detailed derivation is given in Ref. [41] (see also Ref. [42]) yielding an intrinsic contribution to the thermal Hall conductivity given by

$$
\begin{aligned}
\kappa_{x y}^{\mathrm{int}}(\mu) & =\frac{-1}{e^{2} T} \int d \epsilon(\epsilon-\mu)^{2} \sigma_{x y}^{\mathrm{int}}(\epsilon) n_{F}^{\prime}(\epsilon-\mu) \\
& \approx \frac{\pi^{2}}{3} \frac{k_{B}^{2} T}{e^{2}} \sigma_{x y}^{\mathrm{int}}(\mu) .
\end{aligned}
$$

In going to the second line, which shows the WiedemannFranz relation, we have assumed the temperature is low and the chemical potential is not at a singular point (such as at a Dirac node). The geometry dependence of the intrinsic Hall conductivity $\sigma_{x y}^{\text {int }}(\epsilon)$, inherited from that of the Berry curvature $\Omega(\mathbf{k})$ [see Eqs. (14) and (31)], is thus manifested in the thermal Hall conductivity.

To reiterate, as in the case of application of an electric field, the key distinction between the cases of thermal transport considered here is that the perturbing field $T(\mathbf{r})$ varies smoothly in space and the value of this field felt by an electron in orbital $i$ depends on that orbital's spatial position.

\section{Which responses are measured in experiments?}

In both the electrical and thermal transport cases discussed above, we described two types of responses that seem as if they should be very similar, but generally give different results: one giving a geometry independent result and the other giving a geometry dependent result. It is then natural to ask which of these responses is actually measured in an experiment.

(i) For the electrical current response, in order to measure the geometry independent result, one would need to work with neutral fermions so that they do not feel the electromagnetic field produced either by the contacts or by the density of fermions elsewhere in the sample. While this is not the case 
for electrons, a recent experiment with cold fermionic atoms [43] demonstrates that it is possible to measure transport of neutral fermions subjected to applied chemical potential differences between reservoirs. We note that while current densities can depend microscopically on details of the geometry, if one measures the total current across a defined line cutting across a system, this total current must be geometry independent (provided that the pattern of bonds intersected by the cut is held constant, or that the current is evaluated in steady state).

(ii) For most electronic systems, the natural current response to measure will be the usual geometry dependent conductivity $[37,38]$ since the electric field will generally be nonzero. In particular, this implies an intrinsic piece of the response given by Eq. (31). This result is believed to hold very generally at least in the limit $\omega \tau \gg 1$ and $\omega \rightarrow 0$.

(iii) For the case of thermal Hall response, to measure the geometry independent effect, one must be in a temperature regime where phonons are completely decoupled from the device, as we do not want the phonon bath to act as an additional reservoir. We then can attach thermal reservoirs only to the ends of the sample to apply a temperature difference. While such a complete decoupling is only obtainable at extremely low temperatures in most electronic systems, it is quite easily achieved in cold atom experiments. We also note that a system with Coulomb interactions between the fermions will not generally be fully geometry independent since the Coulomb interaction is sensitive to the physical distance between orbitals [i.e., it does not conform to the requirements on interactions discussed around Eq. (7)]. Nonetheless, it may be the case that one can ignore the Coulomb interaction in certain situations so long as no density imbalance builds up.

(iv) To measure the usually considered (and geometry dependent) thermal Hall response one wants to couple to a thermal reservoir throughout the entire sample to impose a uniform gradient. For a two-dimensional system (such as graphene) this could be done by sandwiching the sample between two reservoirs which themselves have thermal gradients. It is also possible that the system's own phonons can act as this reservoir.

\section{Thermal transport in spin systems}

The approach of analyzing (thermal) Hall transport is fairly general and can also be applied to systems with (bosonic) magnon excitations [44-49]. A fairly detailed discussion of this approach is given in Ref. [44]. In such a scheme a band structure is determined for magnons, and the corresponding Bloch eigenstates can be used to calculate a Berry curvature. The thermal Hall conductance is then given by an integral over the Brillouin zone [44,47] (written here in the twodimensional case for simplicity)

$$
\kappa_{x y}=\frac{-k_{B}^{2} T}{\hbar} \int \frac{d^{2} k}{(2 \pi)^{2}} c\left(\omega_{\mathbf{k}}\right) \Omega(\mathbf{k}),
$$

where $c\left(\omega_{\mathbf{k}}\right)$ is a known simple function [44,47] of the frequency of the magnon, $\omega_{\mathbf{k}}$, and $\Omega(\mathbf{k})$ is the Berry curvature. As with the case of the thermal Hall coefficient obtained from electronic band structure in Sec. VI B 2, the derivation of this result assumes coupling to a bath with a uniform thermal gradient. As with that result, this formula is manifestly geometry dependent.

As mentioned in the prior section, while coupling to a thermal bath of uniform thermal gradient may be an accurate representation of certain experiments, it is also possible that thermal transport experiments can be constructed where this is not the case. In particular, if one has a spin system where only the spins carry heat (meaning no phonons or other thermal bath), then energy is transported between sites according to the geometry independent graph of exchange couplings that plays the analogous role to the tight-binding Hamiltonian in Eq. (1). In this case, the thermal transport is in fact geometry independent, and Eq. (32) cannot apply. While spin systems may host long-range dipolar interactions in addition to shortrange exchange terms, these dipolar terms are often very weak and may not be significant for thermal transport. Thus, spin systems are potentially advantageous for observing geometry independent responses.

\section{FURTHER DIRECTIONS AND CONCLUSIONS}

In this work, we have investigated the roles of orbital connectivity and geometry in electronic lattice systems. To expose these concepts, we worked within a tight-binding framework where the information about the physical positions $\left\{\mathbf{r}_{i}\right\}$ of the atomic sites is abstracted away and the electronic dynamics is fully captured by the network of hopping amplitudes and onsite potentials encoded in the tight-binding parameters $\left\{t_{i j}\right\}$. By taking this point of view, insight can be gained about physical quantities which either do or do not depend explicitly on the geometry of the system, as encoded in the positions $\left\{\mathbf{r}_{i}\right\}$. In particular, we studied how the Bloch band Berry curvature and related observables depend on the atomic positions when the tight-binding parameters are kept fixed. These considerations guided us to a refinement of the "geometric stability hypothesis" regarding the stability of fractional Chern insulators, and provided valuable insight into the nature of semiclassical dynamics and various Hall-type transport measurements.

In Sec. VI we examined the Hall response of a noninteracting electron system with a partially filled band. Here, we discussed the different responses that arise when a chemical potential difference is applied between contacts, or when a uniform electric field is applied to the system. We discuss how the former gives a geometry independent response, whereas the latter is geometry dependent. We find a similar situation for the thermal Hall response where one can either apply a thermal difference between reservoirs or a coupling to a uniform thermal gradient over the length of the sample. We commented that similar arguments apply to the thermal Hall response due to magnons. We note in passing that other responses can also be similarly analyzed, such as the thermoelectric Nernst or Peltier responses [42,50].

While we have worked within the tight-binding formulation, our results are more general. In principle, one could imagine altering a spatial metric while at the same time changing the metric for the continuum Schrödinger equation such that the eigenenergies remain unchanged. Similarly, we will discover a change in Berry curvature and our above arguments will remain unchanged. (Another way to understand 
this would be to take a continuum limit of the discrete hopping models that we considered.)

More generally, any calculation can be tested against the backdrop of the type of geometric invariance that we have introduced. As with gauge invariance (or covariance), a calculation that fails to transform correctly must be incorrect at some level. We propose that this approach can be valuable for the field in providing a type of consistency check for a range of results.

In compliance with EPSRC policy framework on research data, this publication is theoretical work that does not require supporting research data.

\section{ACKNOWLEDGMENTS}

The authors acknowledge helpful conversations with $\mathrm{N}$. Lindner, N. Read, B. Doucot, N. Cooper, T. Neupert, A. Bernevig, G. Möller, H. Price, J. Song, and O. Zilberberg. S.H.S. has been supported by the Niels Bohr International Academy, the Simons foundation, and EPSRC Grants No. EP/I031014/1, No. EP/N01930X/1, and No. EP/S020527/1. M.R. gratefully acknowledges the support of the Villum Foundation, and the European Research Council (ERC) under the European Union Horizon 2020 Research and Innovation Programme (Grant Agreement No. 678862).

\section{APPENDIX A: GEOMETRIC STABILITY HYPOTHESIS}

In addition to the Berry curvature, another interesting measure of the "quantum geometry" of a band structure is the so-called Fubini-Study metric, defined as [25,51-55]

$$
g_{\mu \nu}(\mathbf{k})=\frac{1}{2}\left[\left\langle\frac{\partial u}{\partial k_{\mu}} \mid \frac{\partial u}{\partial k_{\nu}}\right\rangle-\mathcal{A}_{\mu} \mathcal{A}_{\nu}\right]+(\mu \rightarrow v),
$$

where both $u$ and $\mathcal{A}$ are functions of $\mathbf{k}$, and $\mu$ and $v$ run over the Cartesian directions of the system. Under a change in geometry as described in Eq. (10) and the resulting Eq. (11), this metric transforms nontrivially. In the case of two-dimensional bands, two additional interesting quantities derived from this metric are $[12,25,27]$ :

$$
\begin{gathered}
D(\mathbf{k})=\operatorname{det} g(\mathbf{k})-\frac{1}{4}|\Omega(\mathbf{k})|^{2} \\
T(\mathbf{k})=\operatorname{Tr} g(\mathbf{k})-|\Omega(\mathbf{k})| .
\end{gathered}
$$

Note that both $D$ and $T$ are zero for a continuum Landau level, and $D$ is also zero for any two-band model $[11,12]$. Further, it was shown [25] that both $T$ and $D$ are non-negative, and that if $T$ is zero everywhere in the Brillouin zone, then $D$ is also.

It was argued that in addition to being favored by flat Berry curvature, large fractional Chern insulator gaps are favored when the Fubini-Study metric $g_{\mu \nu}(\mathbf{k})$ is uniform in the Brillouin zone, as well as having $T$ and $D$ close to zero throughout the zone $[12,25,27,53]$ [see Eqs. (A1)-(A3)]. These three conditions [(i) flatness of Berry curvature $\Omega$, (ii) flatness of $g_{\mu \nu}$, and (iii) minimal values of $D$ and $T$ ] are designed to make the band resemble a continuum Landau level as much as possible. The statement that optimizing these conditions maximizes the many-body gap has been termed the "geometric stability hypothesis" [12] and has been supported in a number of numerical works [12,23,27].

As with the Berry curvature, the Fubini-Study metric, as well as $D$ and $T$, generically change under geometric transformations that leave the hoppings and interaction matrix elements (in the site basis) completely unchanged. Crucially, under such transformations, the many-body gap is unchanged. Thus, the hypothesis cannot be correct as stated. Nonetheless, one cannot discard the observed correlations between gaps and various band parameters [hypotheses (i)-(iii) above] which have been measured in prior numerical works $[12,23,27]$.

We note that in several prior works, the geometry (i.e., the positions of sites in the unit cell) used in numerical simulations happens to correspond to the geometry that minimizes the variance of Berry curvature over the Brillouin zone (i.e., it maximizes the flatness), for a given set of tight-binding parameters. For example, in the fractionally filled Haldane model studied numerically in Refs. [12,23] the minimal variance of the Berry curvature (i.e., the highest "flatness") happens to occur when the orbitals lie on a simple honeycomb, which is the geometry that was considered in Ref. [12]. Similarly, for the Hofstadter model with small flux per plaquette, the Berry curvature is most flat when the orbitals in the magnetic unit cell lie equally spaced, which is exactly the case studied in Ref. [27].

Inspired by this coincidence, we now suggest a possible modification of the geometric stability hypothesis which would be consistent with the geometric invariance required by the arguments in the main text. We propose to measure the variation (the flatness) of the Berry curvature (and Fubini-Study metric) only after optimizing over all possible geometries of the orbitals. In other words, given a particular microscopic model, we accept that the Berry curvature is a function of the geometry of orbitals in the model, but the many-body gap [for Hubbard-type interactions as in Eq. (7)] is not. For a fixed geometry, we measure the variance of the Berry curvature; then we vary over all possible geometries of the orbitals (all positions of the orbitals in the unit cell), while keeping the tight-binding parameters in the Hamiltonian fixed. We then focus on the particular geometry for which the curvature is most flat. We suggest that the many-body gaps will correlate with this optimized quantity. Indeed, this modified conjecture is supported by previous numerical data from Refs. [12,27] which did focus on this optimized orbital geometry. One can apply similar reasoning to the FubiniStudy metric and the resulting quantities $D$ and $T$ as well [Eqs. (A1)-(A3)]. This prescription is well defined, and consistent with the geometric invariance principle. It is a matter for future research to decide if this prescription does indeed predict many-body gaps accurately (and if so, why).

\section{APPENDIX B: EXPLICIT CALCULATIONS OF THE HALL CURRENT RESPONSE}

In this Appendix, we provide two proof-of-principle, explicit Hall transport calculations to support the discussion of geometry dependent and independent responses in the 
main text. For simplicity, we will assume a uniform twodimensional system with noninteracting fermions, in a system free of any disorder. (We take this special case just for demonstration: our qualitative conclusions about geometry dependence and independence do not rely on the absence of disorder.) We also note that here we have not provided any mechanism for electrons to scatter and reequilibrate. Although there are several methods for calculating the Hall response, our approach, in the spirit of Boltzmann theory, will be to consider the dynamics of wave packets in phase space. The particle current density is given by

$$
\mathbf{j}=\int \frac{d \mathbf{k}}{(2 \pi)^{2}} n(\mathbf{k}) \dot{\mathbf{r}}(\mathbf{k})
$$

where $n(\mathbf{k})$ is the occupancy of the state $\mathbf{k}$. From Eq. (20), there are two contributions to $\dot{\mathbf{r}}(\mathbf{k})$ : the regular (i.e., group) velocity $\mathbf{v}_{g}(\mathbf{k})=\nabla_{\mathbf{k}} \varepsilon(\mathbf{k})$, and the anomalous velocity $\mathbf{v}_{a}(\mathbf{k})=$ $\dot{\mathbf{k}} \times \Omega(\mathbf{k})$. In principle, $n(\mathbf{k})$ and $\dot{\mathbf{r}}(\mathbf{k})$ may be functions of position $\mathbf{r}$, giving a current density $\mathbf{j}(\mathbf{r})$ that is a function of position as well. In the presence of inhomogeneities, one may also obtain magnetization currents [5]. However, the simple demonstrations here avoid this added complexity since we have chosen to consider cases which are spatially uniform.

\section{Applied electric field, clean limit}

We consider an infinitely large (or periodic), disorder-free system. We will apply a weak time-dependent uniform electric field $\mathbf{E}(t)$ at low frequency. The system starts in a thermal occupation $n^{(0)}(\mathbf{k})=n_{F}[\varepsilon(\mathbf{k})-\mu]$, and we adiabatically turn on the electric field. ${ }^{9}$ In this case, the $\mathbf{k}$ states simply accelerate freely via $\dot{\mathbf{k}}=-e \mathbf{E}(t)$, as in Eq. (21), without scattering or relaxing. The occupation distribution therefore adiabatically evolves as $n(\mathbf{k}, t)=n_{F}(\varepsilon[\mathbf{k}(t)])$, with the time-dependent wave vector

$$
\mathbf{k}(t)=\mathbf{k}-e \int_{-\infty}^{t} d t^{\prime} \mathbf{E}\left(t^{\prime}\right) .
$$

Using Eq. (B1), the resulting current density is given by

$$
\mathbf{j}(t)=\int \frac{d \mathbf{k}}{(2 \pi)^{2}} n_{F}(\varepsilon[\mathbf{k}(t)]-\mu) \dot{\mathbf{r}}(\mathbf{k}) .
$$

In the absence of $\mathbf{E}$, the current density in the uniform system must vanish everywhere. We are interested in the effects of $\mathbf{E}$ at linear order. There are two places in Eq. (B3) where $\mathbf{E}$ enters: (i) in the anomalous velocity part of $\dot{\mathbf{r}}, \mathbf{v}_{a}(\mathbf{k})$, and (ii) in $\mathbf{k}(t)$. We will consider these two contributions separately, to linear order in $\mathbf{E}$. To stay in the linear response regime, we therefore assume that the frequency is low enough to be in the adiabatic limit with respect to interband transitions, while the electric field is also small enough that $e|\mathbf{E}| / \omega$ is small enough that nonlinear contributions in $\mathbf{E}$ can be neglected. Such nonlinear corrections (of order $\mathbf{E}^{2} / \omega$ ) are discussed, for example,

\footnotetext{
${ }^{9}$ Although the system may be gapless, there is still a notion of adiabaticity since, in the absence of disorder and interactions, momentum conservation forbids any transitions to other low-energy states in linear response. For small $\mathbf{E}$ and $\omega$, interband transitions are also suppressed.
}

in Ref. [56]. Note that in cases where there is an equilibration mechanism, such as phonons or electron-electron interaction, the current calculation only remains correct so long as the timescale for this equilibration is much longer than $1 / \omega$.

Considering contribution (i) defined above, in linear response we obtain a contribution to the Hall current density from the anomalous velocity term given by

$$
\mathbf{j}_{H}^{0}(t)=e \mathbf{E}(t) \times \hat{\mathbf{z}} \int \frac{d \mathbf{k}}{(2 \pi)^{2}} \Omega(\mathbf{k}) n^{(0)}(\mathbf{k})
$$

where $\hat{\mathbf{z}}$ is the normal to the plane. This thus gives a lowfrequency Hall conductivity of the form in Eq. (31).

We now consider contribution (ii). Here, Eq. (B2) gives the dependence of $\mathbf{k}(t)$ on $\mathbf{E}$. Expanding for small $\mathbf{E}$ and using $\mathbf{v}_{g}(\mathbf{k})=\nabla_{\mathbf{k}} \varepsilon(\mathbf{k})$, for Eq. (B3) we obtain

$$
\varepsilon[\mathbf{k}(t)]=\varepsilon(\mathbf{k})-e \mathbf{v}_{g}(\mathbf{k}) \cdot \int_{-\infty}^{t} d t^{\prime} \mathbf{E}\left(t^{\prime}\right)+\cdots
$$

Substituting this expression into Eq. (B3) and expanding to linear order in $\mathbf{E}$ we obtain a contribution to the current density

$$
\begin{aligned}
& \delta j_{\mu}(t) \\
& \quad=-e \int_{-\infty}^{t} d t^{\prime} E_{v}\left(t^{\prime}\right) \int \frac{d \mathbf{k}}{(2 \pi)^{2}} v_{g, \mu}(\mathbf{k}) v_{g, v}(\mathbf{k}) n_{F}^{\prime}(\varepsilon(\mathbf{k})-\mu),
\end{aligned}
$$

where $\mu, v$ label the Cartesian directions. Crucially, $\delta j_{\mu}(t)$ gives no Hall component of the response, as the $\mathbf{k}$ integral on the right-hand side is symmetric between $\mu$ and $\nu$. (Note that one must take only the antisymmetric component $\sigma_{x y}-\sigma_{y x}$ to isolate the Hall component.) Thus, the Hall current density remains that given by Eq. (B4). This response is geometry dependent due to the geometry dependence of $\Omega(\mathbf{k})$.

\section{Chemical potential difference, clean limit}

Here, we consider the case of a system of neutral fermions being coupled to two reservoirs at different chemical potentials $\mu_{1}$ and $\mu_{2}$. In this case, the dynamics are fully determined by the hopping of particles between sites of the unperturbed tight-binding network, and the response of the system must be geometry independent.

The situation we envisage is inspired by the recent experiments in Ref. [43]. We assume that the reservoirs are separated in the $\hat{\mathbf{x}}$ direction; it is most convenient to think about the sample as being periodic in the $\hat{\mathbf{y}}$ direction and infinitely long in the $\hat{\mathbf{x}}$ direction so that we are considering an infinitely long cylinder all constructed of the same material (the same hopping model). Initially, we split the cylinder into three disconnected pieces: $x<0$ the left reservoir, $0<x<L$ the system, and $x>L$ the right reservoir. We fill the left and right reservoirs with fermions such that $\mu_{1} \neq \mu_{2}$. Then, at some time we connect the three systems together, and we let current flow between the reservoirs. In the long-time limit there will be a steady-state current flow in the system, which 
we measure. The steady-state current flow will be independent of details such as the initial state in the system section $0<x<L$, and the precise procedure for connecting up the three pieces.

In the clean limit, where there is no scattering, transport between the two reservoirs is essentially ballistic. Since there is no electric field, there is no anomalous velocity. The total current density within the bulk is then

$$
\mathbf{j}=\int \frac{d \mathbf{k}}{(2 \pi)^{2}} \nabla_{\mathbf{k}} \varepsilon(\mathbf{k}) n(\mathbf{k}),
$$

where the distribution is given by

$$
n(\mathbf{k})= \begin{cases}n_{F}\left[\varepsilon(\mathbf{k})-\mu_{1}\right], & v_{x}(\mathbf{k})>0 \\ n_{F}\left[\varepsilon(\mathbf{k})-\mu_{2}\right], & v_{x}(\mathbf{k})<0 .\end{cases}
$$

If one considers a dispersion $\varepsilon(\mathbf{k})$ which is symmetric under $k_{y} \leftrightarrow-k_{y}$, the Hall current density will be zero, independent of the Berry curvature of the system's bands and of the orbital geometry. For a less symmetric dispersion, the result may be nonzero (but still independent of the Berry curvature).
[1] M. Z. Hasan and C. L. Kane, Rev. Mod. Phys. 82, 3045 (2010).

[2] X.-L. Qi and S.-C. Zhang, Rev. Mod. Phys. 83, 1057 (2011).

[3] B. Yan and C. Felser, Annu. Rev. Condens. Matter Phys. 8, 337 (2017).

[4] A. Bansil, H. Lin, and T. Das, Rev. Mod. Phys. 88, 021004 (2016).

[5] D. Xiao, M.-C. Chang, and Q. Niu, Rev. Mod. Phys. 82, 1959 (2010).

[6] E. J. Bergholtz and Z. Liu, Int. J. Mod. Phys. B 27, 1330017 (2013).

[7] S. A. Parameswaran, R. Roy, and S. L. Sondhi, C. R. Phys. 14, 816 (2013).

[8] F. D. M. Haldane, Berry's phase and Hilbert space geometry as a new ingredient in Fermi liquid theory, talk presented at the Low-D Quantum Condensed Matter summer workshop at the Center for Mathematical Physics Amsterdam, Netherlands, 2005 (unpublished).

[9] F. D. M. Haldane, arXiv:1401.0529.

[10] L.-K. Lim, J.-N. Fuchs, and G. Montambaux, Phys. Rev. A 92, 063627 (2015).

[11] E. Dobardžić, M. Dimitrijević, and M. V. Milovanović, Phys. Rev. B 89, 235424 (2014).

[12] T. S. Jackson, G. Möller, and R. Roy, Nat. Commun. 6, 8629 (2015).

[13] N. R. Cooper, J. Dalibard, and I. B. Spielman, Rev. Mod. Phys. 91, 015005 (2019).

[14] M. Fruchart, D. Carpentier, and K. Gawedzki, Europhys. Lett. 106, 60002 (2014).

[15] A. Kol and N. Read, Phys. Rev. B 48, 8890 (1993).

[16] A. S. Sørensen, E. Demler, and M. D. Lukin, Phys. Rev. Lett. 94, 086803 (2005).

[17] M. Hafezi, A. S. Sørensen, E. Demler, and M. D. Lukin, Phys. Rev. A 76, 023613 (2007).

[18] G. Möller and N. R. Cooper, Phys. Rev. Lett. 103, 105303 (2009).

[19] T. Neupert, L. Santos, C. Chamon, and C. Mudry, Phys. Rev. Lett. 106, 236804 (2011).

[20] E. Tang, J.-W. Mei, and X.-G. Wen, Phys. Rev. Lett. 106, 236802 (2011).

[21] K. Sun, Z. Gu, H. Katsura, and S. Das Sarma, Phys. Rev. Lett. 106, 236803 (2011).

[22] N. Regnault and B. A. Bernevig, Phys. Rev. X 1, 021014 (2011).

[23] Y.-L. Wu, B. A. Bernevig, and N. Regnault, Phys. Rev. B 85, 075116 (2012).

[24] Y.-F. Wang, Z.-C. Gu, C.-D. Gong, and D. N. Sheng, Phys. Rev. Lett. 107, 146803 (2011).
[25] R. Roy, Phys. Rev. B 90, 165139 (2014).

[26] M. Udagawa and E. J. Bergholtz, J. Stat. Mech.: Theory Exp. (2014) P10012.

[27] D. Bauer, T. S. Jackson, and R. Roy, Phys. Rev. B 93, 235133 (2016).

[28] C. H. Lee, M. Claassen, and R. Thomale, Phys. Rev. B 96, 165150 (2017).

[29] An interesting possibility, suggested by Ref. [11], is that one should focus on special points in the Brillouin zone where the Berry curvature is independent of geometric transformation. This appears possible for two-band models only.

[30] M.-C. Chang and Q. Niu, Phys. Rev. B 53, 7010 (1996).

[31] H. M. Price and N. R. Cooper, Phys. Rev. A 85, 033620 (2012).

[32] G. Jotzu, M. Messer, R. Desbuquois, M. Lebrat, T. Uehlinger, D. Greif, and T. Esslinger, Nature (London) 515, 237 (2014).

[33] M. Aidelsburger, M. Lohse, C. Schweizer, M. Atala, J. T. Barreiro, S. Nascimbène, N. R. Cooper, I. Bloch, and N. Goldman, Nat. Phys. 11, 162 (2015).

[34] N. Flaschner, B. S. Rem, M. Tarnowski, D. Vogel, D.-S. Luhmann, K. Sengstock, and C. Weitenberg, Science 352, 1091 (2016).

[35] M. Wimmer, H. M. Price, I. Carusotto, and U. Peschel, Nat. Phys. 13, 545 (2017).

[36] K. Wintersperger, C. Braun, F. N. Unal, A. Eckardt, M. Di Liberto, N. Goldman, I. Bloch, and M. Aidelsburger, Nat. Phys. 16, 1058 (2020).

[37] N. Nagaosa, J. Sinova, S. Onoda, A. H. MacDonald, and N. P. Ong, Rev. Mod. Phys. 82, 1539 (2010).

[38] N. A. Sinitsyn, J. Phys.: Condens. Matter 20, 023201 (2008).

[39] P. Nozières and C. Lewiner, J. Phys. (Paris) 34, 901 (1973).

[40] V. K. Dugaev, P. Bruno, M. Taillefumier, B. Canals, and C. Lacroix, Phys. Rev. B 71, 224423 (2005).

[41] T. Qin, Q. Niu, and J. Shi, Phys. Rev. Lett. 107, 236601 (2011).

[42] F. D. M. Haldane, Phys. Rev. Lett. 93, 206602 (2004).

[43] S. Krinner, D. Stadler, D. Husmann, J.-P. Brantut, and T. Esslinger, Nature (London) 517, 64 (2015).

[44] R. Matsumoto and S. Murakami, Phys. Rev. B 84, 184406 (2011).

[45] H. Katsura, N. Nagaosa, and P. A. Lee, Phys. Rev. Lett. 104, 066403 (2010).

[46] H. Lee, J. H. Han, and P. A. Lee, Phys. Rev. B 91, 125413 (2015).

[47] R. Matsumoto and S. Murakami, Phys. Rev. Lett. 106, 197202 (2011).

[48] Y. H. Gao and G. Chen, SciPost Phys. Core 2, 004 (2020). 
[49] A. Mook, J. Henk, and I. Mertig, Phys. Rev. B 89, 134409 (2014).

[50] D. Xiao, Y. Yao, Z. Fang, and Q. Niu, Phys. Rev. Lett. 97, 026603 (2006).

[51] N. Marzari and D. Vanderbilt, Phys. Rev. B 56, 12847 (1997).

[52] T. Neupert, C. Chamon, and C. Mudry, Phys. Rev. B 87, 245103 (2013).

[53] E. Dobardžić, M. V. Milovanović, and N. Regnault, Phys. Rev. B 88, 115117 (2013).
[54] T. Ozawa and N. Goldman, Phys. Rev. B 97, 201117(R) (2018).

[55] M. Yu, P. Yang, M. Gong, Q. Cao, Q. Lu, H. Liu, S. Zhang, M. B. Plenio, F. Jelezko, T. Ozawa, N. Goldman, and J. Cai, Natl. Sci. Rev. 7, 254 (2019).

[56] I. Sodemann and L. Fu, Phys. Rev. Lett. 115, 216806 (2015).

[57] N. A. Sinitsyn, Q. Niu, J. Sinova, and K. Nomura, Phys. Rev. B 72, 045346 (2005). 